\title{
Transient Global Amnesia following Vertebral Artery Angioplasty and Stenting
}

\author{
Hahn Young Kim ${ }^{\text {a,d }}$ Hyun-Seung Kang ${ }^{b}$ Hong-Gee Roh ${ }^{c}$ Jeeyoung Oh ${ }^{a} \quad$ II-Keun Lee $^{a}$ \\ Seol-Heui Han a, d \\ Departments of a Neurology, ${ }^{b}$ Neurosurgery and ${ }^{\mathrm{c}}$ Radiology, and ${ }^{\mathrm{d} C e n t e r ~ f o r ~ G e r i a t r i c ~ N e u r o s c i e n c e ~ R e s e a r c h, ~}$ \\ Konkuk University School of Medicine, Seoul, South Korea
}

Dear Sir,

Transient global amnesia (TGA) is a sudden-onset clinical syndrome characterized by a loss of memory for recent events and an inability to retain new memories [1]. Diverse mechanisms have been proposed as possible etiologies, including cerebral ischemia, arterial vasospasm, cerebral venous congestion, epilepsy and $\mathrm{mi}$ graine [2-7]. A tiny ischemic lesion in the temporal lobe, hippocampus or parahippocampal area is one possible cause [3]. Angioplasty with stenting of the posterior circulation is becoming a widely available neurointervention in patients with stenosis of the vertebral or basilar artery. We describe a patient with TGA following angioplasty with stenting of the vertebral artery.
Case Report

A 62-year-old right-handed man noted recurrent episodes of transient diplopia with vertigo for $10 \mathrm{~min}$. He had stroke risk factors of hypertension and a smoking history of 35 pack-years. He reported no previous history of migraine or seizure. No responsible lesion was detected on diffusionweighted MRI performed on the day of
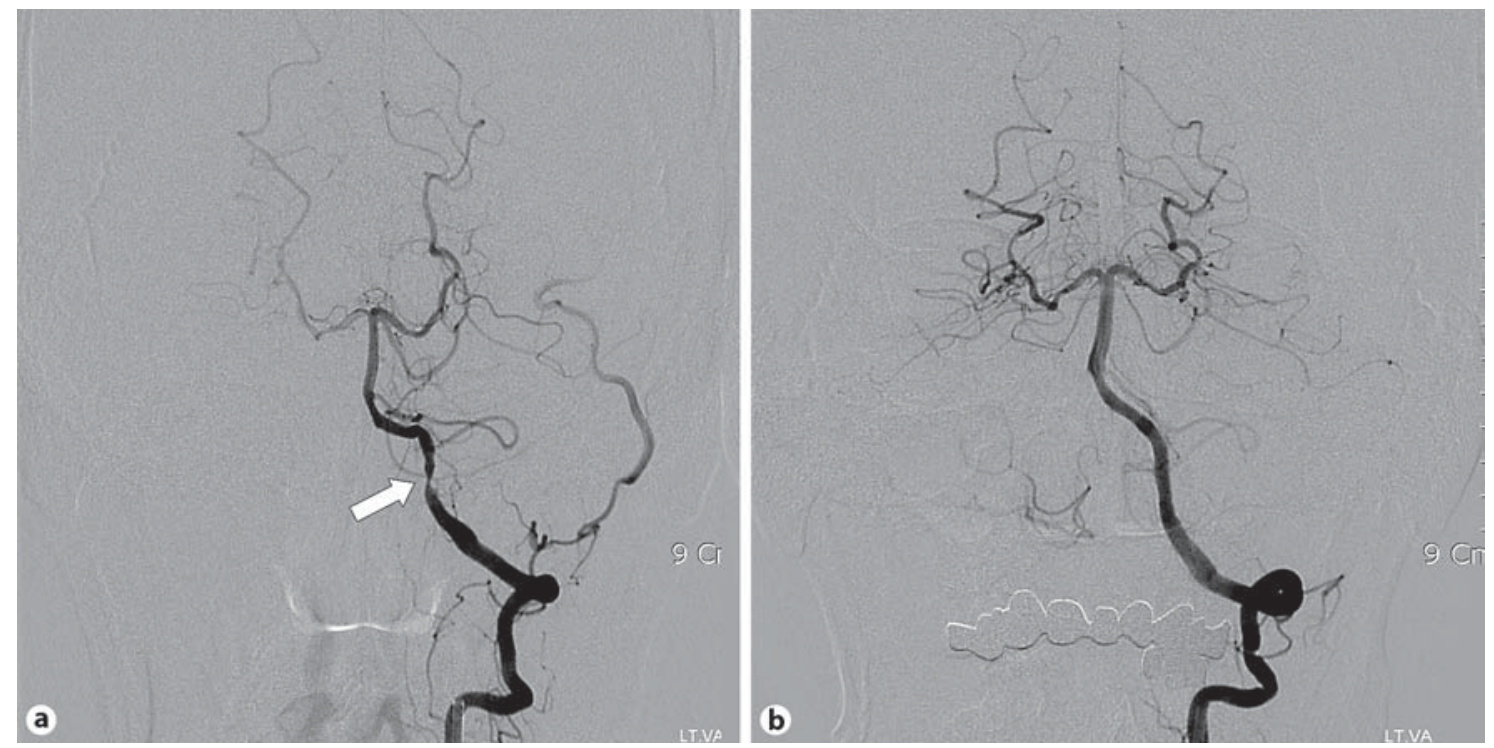

Fig. 1. Conventional angiography revealed severe stenosis of the left vertebral artery (white arrow in a), which was revascularized by successful angioplasty and stenting (b).

\section{KARGER}

Fax +41613061234 E-Mail karger@karger.ch www.karger.com
(C) 2006 S. Karger AG, Basel

$0014-3022 / 06 / 0562-0133 \$ 23.50 / 0$

Accessible online at: www.karger.com/ene
Seol-Heui Han, MD

4-12 Hwayang-dong

Gwangjin-gu

Seoul 143-729 (South Korea)

Tel. +82 22030 7561, Fax +82 22030 5169, E-Mail alzdoc@kuh.ac.kr 

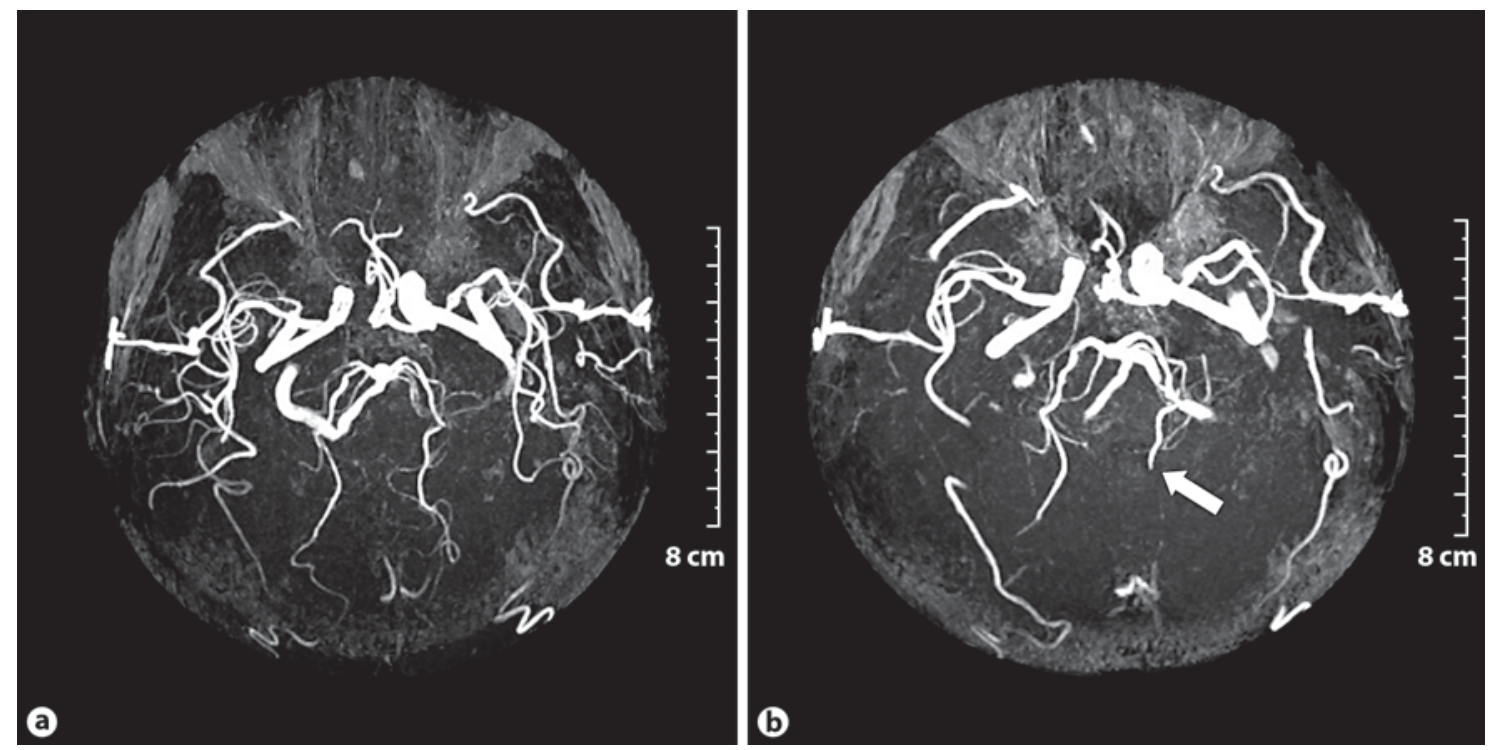

Fig. 2. MRA immediately after the onset of TGA showed abrupt signal loss in the left posterior cerebral artery (white arrow in b), which had been visible on the initial MRA (a).
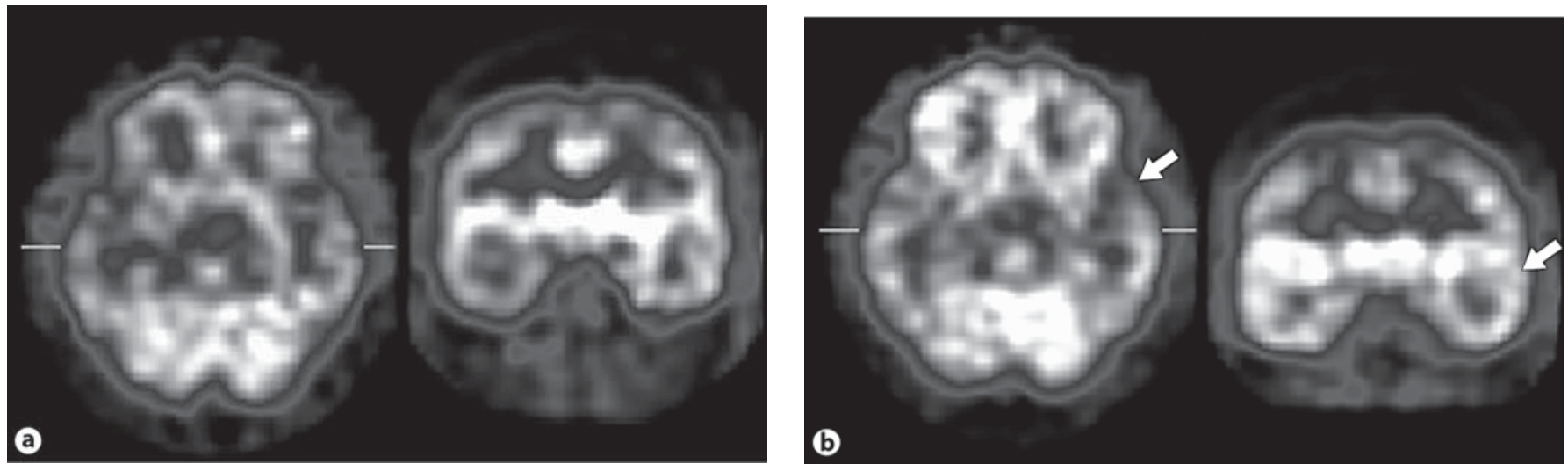

Fig. 3. Baseline (a) and postacetazolamide SPECT scan (b) performed 5 days after the TGA, showing mild hypoperfusion of the left temporal area (white arrows in $\mathbf{b}$ ).

symptom onset. Magnetic resonance angiography (MRA) revealed that severe stenosis of the left vertebral artery was responsible for his transient ischemic attack.

Selective cerebral angiography was performed using iodixanol (Visipaque, Amersham Health, Cork, Ireland; fig. 1a). Vertebral angioplasty with stenting was performed successfully (fig. 1b). No vasospasm or dissection was observed during the procedure.

After the procedure, the patient became perplexed and could not remember having had an angiography. He did not even know he was scheduled to undergo angiography. He repeatedly asked where he was and why he was in hospital. His attention was normal, but his anterograde memory was impaired. No other focal neurological symptom or sign was apparent on neurological examination. His visual field was also intact.

To exclude an acute ischemic lesion, diffusion-weighted MRI (DWI) was performed $1 \mathrm{~h}$ after the onset of the amnestic event. No responsible lesion was identified. However, signal loss in the distal part of the left posterior cerebral artery was ob- served on MRA, although it had been visible on the initial MRA (fig. 2).

The patient's anterograde amnesia improved, and the results of a 3-word registration and recall test were normal within the next $24 \mathrm{~h}$. The period of memory lapse gradually decreased over the next 3 days, but his failure to recall the whole angioplasty procedure was permanent.

${ }^{99 \mathrm{~m}}$ Tc-HMPAO single-photon emission computed tomography (SPECT) performed 5 days after the amnestic event revealed mild hypoperfusion in the left temporal area (fig. 3). A neuropsycholog- 
ical test performed 7 days after the amnestic event revealed mild impairment of the verbal memory, although the results of a visual memory test were normal (Seoul Verbal Learning Test: immediate recall, 55th percentile; delayed recall, 18th percentile; recognition score, 14 th percentile vs. Rey Complex Figure Test: immediate recall, 29th percentile; delayed recall, 43rd percentile; recognition score 95th percentile). The patient's attention, language and visuospatial functions were normal. He was placed on a medication regimen of clopidogrel and aspirin and remained asymptomatic at the 6-month follow-up.

\section{Discussion}

Of the various alternatives, cerebrovascular ischemia is one of the most probable mechanisms underlying TGA, when it is accompanied by other neurological symptoms or stroke risk factors [4]. Tiny lesions visible on DWI, mainly in the region of the posterior cerebral artery, have been revealed $[3,5]$. Some reports maintained that no responsible lesion was visible on DWI $[6,7]$. However, in the recent literature, very small lesions invisible in the hyperacute phase have been visualized later in the follow-up study with an interval of 24-48 h after the onset of TGA $[8,9]$. If a follow-up DWI study had been performed in our case, a responsible lesion might have been revealed. DWI performed with an interval of only $1 \mathrm{~h}$ might be too early to visualize tiny lesions.

TGA associated with coronary angiography and angioplasty has been reported [10]. These reports might share some causal factor with our case. Several cases of TGA associated with cerebral angiographic procedures with contrast dye have also been reported $[4,11,12]$. In these patients, arterial vasospasm or direct neurotoxicity induced by the contrast dye was implicated as a possible cause. Ionic contrast dye was the culprit in some cases [11]. After the ionic contrast dye had been replaced by a nonionic dye, the incidence of TGA decreased [4, 12]. The slightly higher temperature of the non- ionic contrast dye at the time of injection has also been implicated [4]. We used nonionic contrast dye prewarmed to body temperature. Temporal lobe epilepsy or regional brain dysfunction related to the disturbed venous outflow patterns should be considered as another possible cause $[13,14]$.

The abrupt signal loss in the left posterior cerebral artery may imply an embolic occlusion. The blood flow in the hippocampal artery, which branches from the posterior cerebral artery, may also have decreased. Although no responsible lesion was identified on DWI, mild hypoperfusion of the left temporal area on brain SPECT and predominantly verbal memory impairment, compared with his unaffected visual memory, implied left temporal lobe dysfunction. In some cases of TGA, hypoperfusion in the temporal area was observed on brain SPECT performed 1 week after symptom onset $[3,15]$. Mild abnormalities in neuropsychological test results, indicating left temporal dysfunction, might imply sustained hypoperfusion or diaschisis in the left temporal area, although the clinical symptoms were completely eliminated.

In summary, arterioarterial embolism of the posterior cerebral artery is the most probable mechanism underlying TGA in our patient, although no lesion was detected on DWI. TGA might be considered one of the rare complications of vertebral angioplasty with stenting, albeit with a favorable prognosis.

\section{Acknowledgment}

This paper was supported by Konkuk University in 2006.

\section{References}

1 Fisher CM, Adams RD: Transient global amnesia. Acta Neurol Scand 1964;40:1-83.

2 Hodges JR, Warlow CP: The aetiology of transient global amnesia: a case-control study of 114 cases with prospective followup. Brain 1990;113:639-657.

3 Jeong Y, Kim GM, Min Song Y, Na DL: A tiny hippocampal ischemic lesion associated with transient global amnesia. Cerebrovasc Dis 2003;16:439-442.
4 Jackson A, Stewart G, Wood A, Gillespie JE: Transient global amnesia and cortical blindness after vertebral angiography: further evidence for the role of arterial spasm. Am J Neuroradiol 1995; 16:955-959.

5 Woolfenden AR, O'Brien MW, Schwartzberg RE, Norbash AM, Tong DC: Diffusionweighted MRI in transient global amnesia precipitated by cerebral angiography. Stroke 1997;28:2311-2314.

6 Gass A, Gaa J, Hirsch J, Schwartz A, Hennerici MG: Lack of evidence of acute ischemic tissue change in transient global amnesia on single-shot echo-planar diffusion-weighted MRI. Stroke 1999;30:2070-2072.

7 Huber R, Aschoff AJ, Ludolph AC, Riepe MW: Transient global amnesia: evidence against vascular ischemic etiology from diffusion-weighted imaging. J Neurol 2002; 249:1520-1524.

8 Sedlaczek O, Hirsch JG, Grips E, Peters CN, Gass A, Wohrle J, Hennerici M: Detection of delayed focal MR changes in the lateral hippocampus in transient global amnesia. Neurology 2004;62:2165-2170.

9 Felix MM, Castro LH, Maia AC Jr, da Rocha AJ: Evidence of acute ischemic tissue change in transient global amnesia in magnetic resonance imaging: case report and literature review. J Neuroimaging 2005;15:203-205.

10 Wong E, Patel A, Jayasinghe R: Transient global amnesia following coronary angiography and angioplasty. Intern Med J 2005;35: 435-436.

11 Wales LR, Nov AA: Transient global amnesia: complication of cerebral angiography. Am J Neuroradiol 1981;2:275-277.

12 Schamschula RG, Soo MY: Transient global amnesia following cerebral angiography with nonionic contrast medium. Australas Radiol 1994;38:196-198.

13 Melo TP, Ferro JM, Paiva T: Are brief or recurrent transient global amnesias of epileptic origin? J Neurol Neurosurg Psychiatry 1994;57:622-625

14 Sander D, Winbeck K, Etgen T, Knapp R, Klingelhofer J, Conrad B: Disturbance of venous flow patterns in patients with transient global amnesia. Lancet 2000;356:19821984.

15 Jovin TG, Vitti RA, McCluskey LF: Evolution of temporal lobe hypoperfusion in transient global amnesia: a serial single-photon emission computed tomography study. J Neuroimaging 2000;10:238-241. 